\title{
Stabilization of an Edentulous Premaxilla for an Alveolar Bone Graft: Case Report
}

\author{
Paul Bohman, D.D.S. \\ DenNis Duke Yamashita D.D.S. \\ Seung-Hak Baek, D.D.S., Ph.D. \\ StePHEN L.-K. Yen, D.M.D., Ph.D.
}

\begin{abstract}
Objective: The patient with bilateral cleft lip and palate and an edentulous premaxilla poses several additional challenges to the surgeon and the orthodontist. The cleft segments are usually expanded and stabilized with toothsupported orthodontic appliances prior to an alveolar bone graft. This report describes a technique for stabilizing an edentulous premaxilla using an orthodontic-surgical screw that is ligated to an orthodontic archwire.
\end{abstract}

KEY WORDS: edentulous premaxilla, orthodontics, secondary bone grafting

In the treatment of patients with cleft lip and palate, the secondary bone graft is used to provide bony continuity between clefted maxillary segments, provide bone for tooth eruption, support the nose, and maintain the position of expanded maxillary segments (Boyne and Sands, 1972; Abyholm et al., 1981; Dempf et al., 2002). The patient with bilateral cleft lip and palate with an edentulous premaxilla poses several additional challenges to the surgeon and orthodontist. The usual stabilization of the three maxillary segments with orthodontic archwires and expanders is not possible because the edentulous premaxilla does not contain teeth that can be used for bracket attachment and wire fixation. Similarly, surgical splints would not be able to immobilize a small edentulous premaxilla. Premaxillary excision would create a larger defect and require a more complex bone graft or segmental distraction to minimize the size of the defect. In this report, a technique for stabilizing an edentulous premaxilla using an orthodontic-surgical screw that is ligated to an orthodontic archwire is presented.

\section{Case History}

A 34-year-old woman with bilateral cleft lip and palate presented to the Oral and Maxillofacial Surgery Division at Los Angeles County/University of Southern California Medical

Dr. Bohman is in private practice in Los Angeles, California, and is former chief resident, Department of Oral and Maxillofacial Surgery, University of Southern California. Dr. Yamashita is Chairman, Department of Oral and Maxillofacial Surgery, University of Southern California. Dr. Baek is a visiting scholar and currently with the Department of Orthodontics, College of Dentistry, Seoul National University, Yeonkundong, Jongroku, 110-744, Seoul, Korea. Dr. Yen is a Staff Orthodontist, USC/Los Angeles County Hospital and Childrens Hospital Los Angeles and Assistant Professor, University of Southern California.

Submitted January 2003; Accepted May 2003.

Address correspondence to: Stephen L.-K. Yen, D.M.D., Ph.D., Department of Oral and Maxillofacial Surgery, Los Angeles County/University of Southern California Medical Center, OPD, IP51, 2010 Zonal Avenue, Los Angeles, California 90033.E-mail syen@usc.edu.
Center for treatment beyond her primary cleft lip and palate repair. She had had no surgery since her primary repair in Ecuador as an infant. On clinical examination, her maxillary segments were collapsed and severely constricted. She had anterior and bilateral posterior cross bites with a skeletal Class III malocclusion resulting from maxillary hypoplasia. Occlusal and panoramic radiographs revealed that her premaxilla was small (measuring only $8 \mathrm{~mm}$ in the anterior-posterior dimension), bulb shaped, and edentulous (Fig 1A through 1E).

The orthodontic preparation for the planned bone graft consisted of expansion of the lateral segments using a quadhelix appliance. In preparation for a future LeFort 1 maxillary advancement, the endpoint of the expansion was the restoration of arch form and an arch width that coordinated with the mandibular arch. After expansion, the bulbous premaxilla was attached to a narrow stalk of bone. The premaxilla was mobile and could be moved $5 \mathrm{~mm}$ in any direction with light finger pressure. Labial orthodontic brackets were bonded to the molars, premolars, and canines to support a $0.018^{\prime \prime} \times 0.018^{\prime \prime}$ stainless steel archwire. Ordinarily a tooth in the premaxilla would have been used to immobilize the segment with a rectangular archwire and edgewise bracket. To stabilize the premaxilla for an alveolar bone graft, a surgical-orthodontic screw was substituted for the dental tooth and root. The head of the surgical screw contained an edgewise rectangular orthodontic archwire slot that could engage a rectangular archwire. At the time of surgery (Fig. 2A through C; screw provided by Epoch Medical, Seoul, Korea), the bracket slot and wire were engaged and ligated so that the premaxilla could not be moved by hand pressure.

Under nasotracheal intubation and general anesthesia, the lateral segment of the left cleft site was approached using an anteriorly based buccal rotation finger flap and division of the palatal mucosa into a nasal and oral reflection. A vertical incision was made on the premaxillary segment to create a labial and palatal mucosal margin (Fig. 2D and 2E). Exposure of the cleft site revealed a sizable $1-\mathrm{cm}$ osseous defect under the 

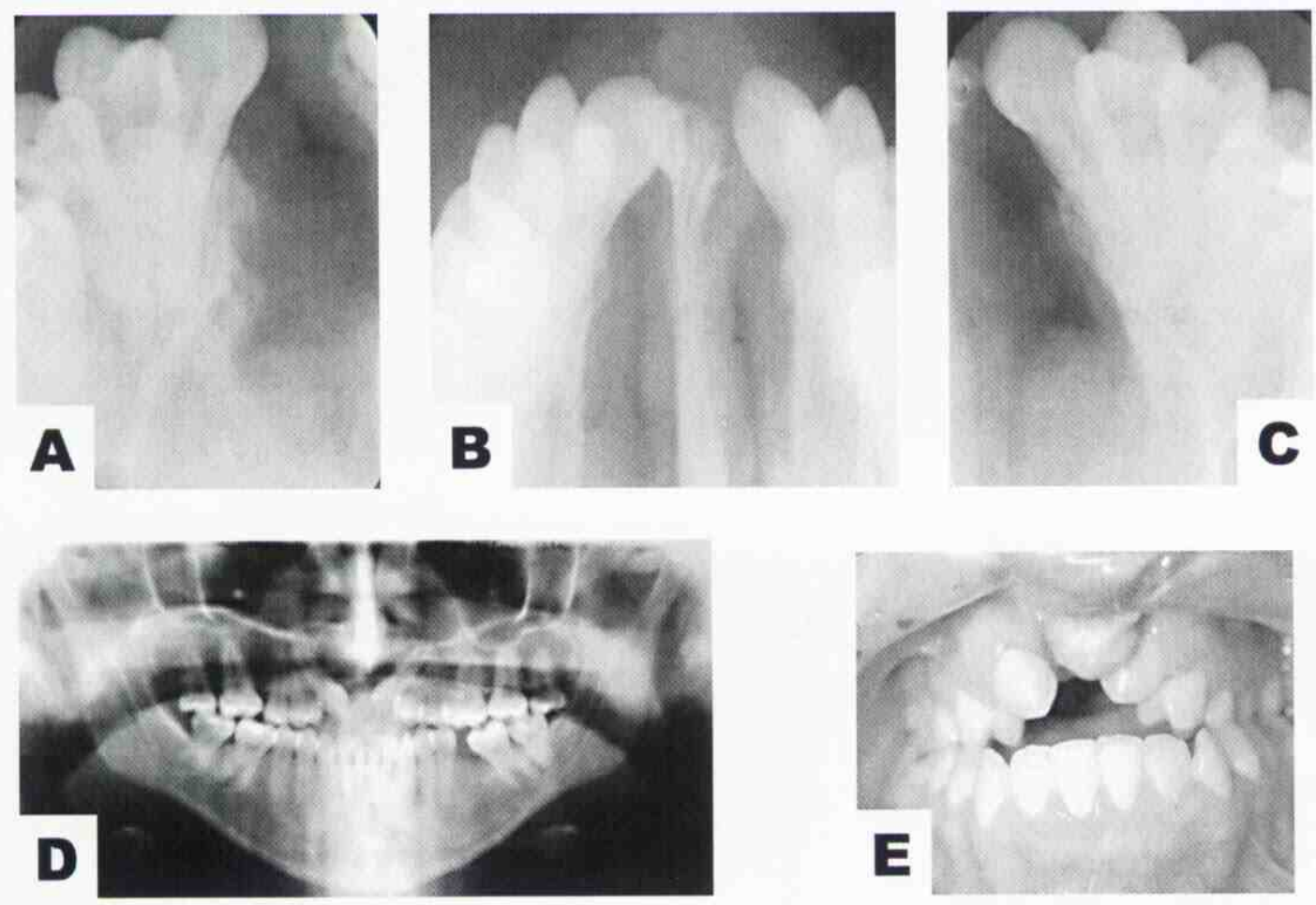

FIGURE 1. Presurgical radiographs and photographs. A: Right periapical radiograph showing cleft defect B: Occlusal radiograph showing edentulous premaxilla. C: Left periapical radiograph showing cleft defect. D: Panoramic radiograph taken before treatment. E: Anterior dental photograph showing premaxillary position and collapsed lateral segments.
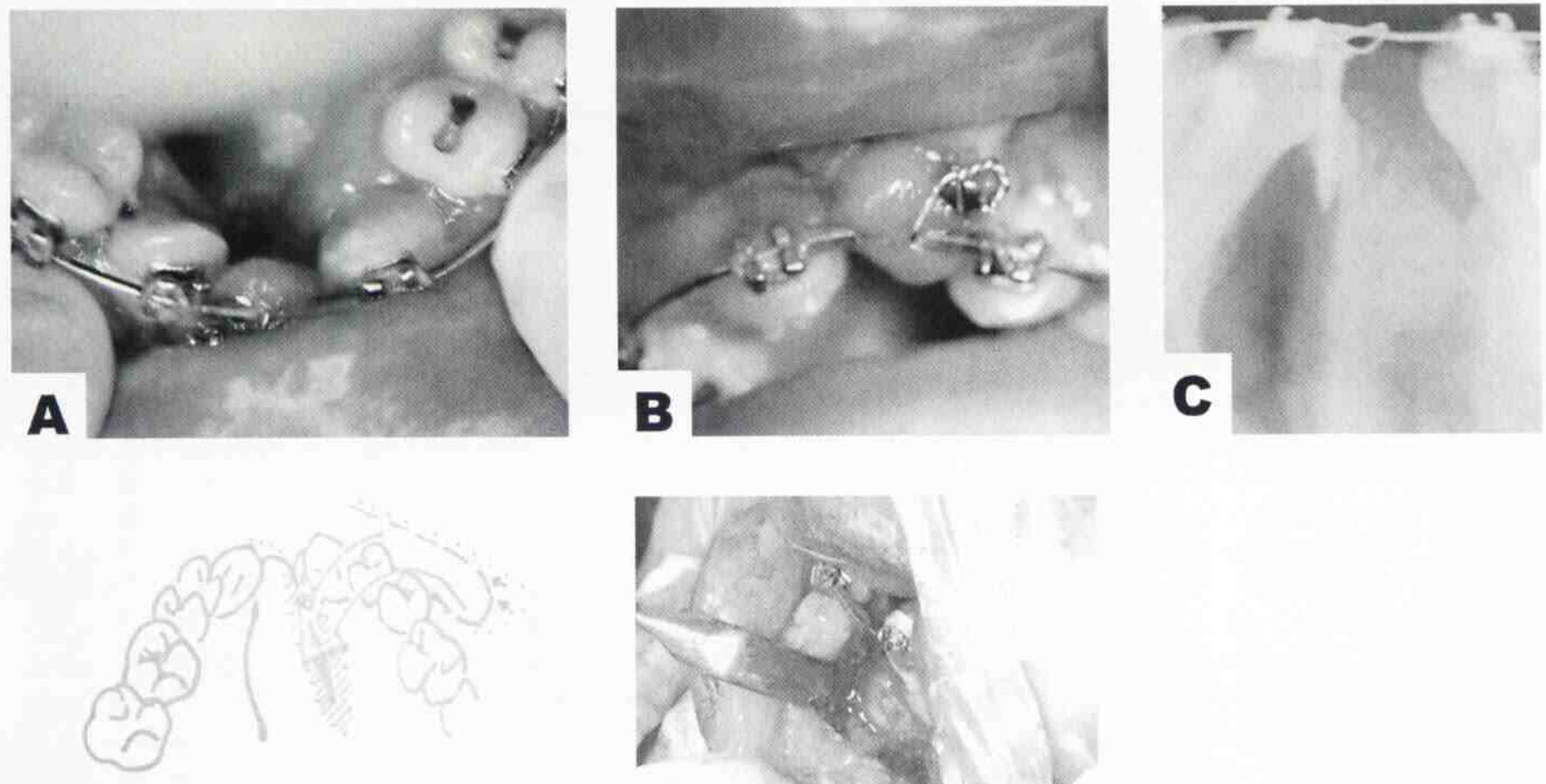

D

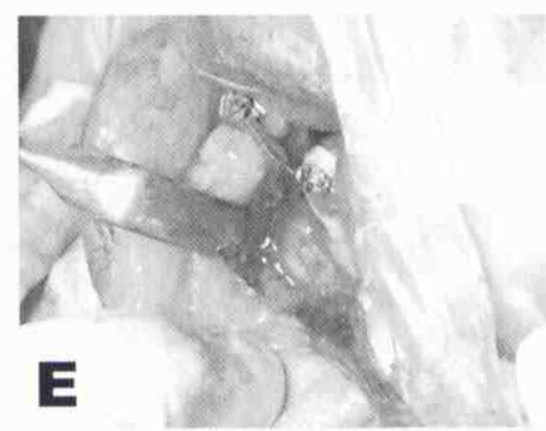

FIGURE 2. Alveolar bone graft for the left side. A: Occlusal photograph showing arch alignment. B: Anterior dental photograph showing placement of screw and ligation to archwire at time of surgery. C: Three-month postoperative radiograph showing bone fill of left alveolar graft. D: Diagram of surgery with arrow to illustrate how flap was rotated to close one side of the cleft. E: Surgical photograph showing cleft defect. 

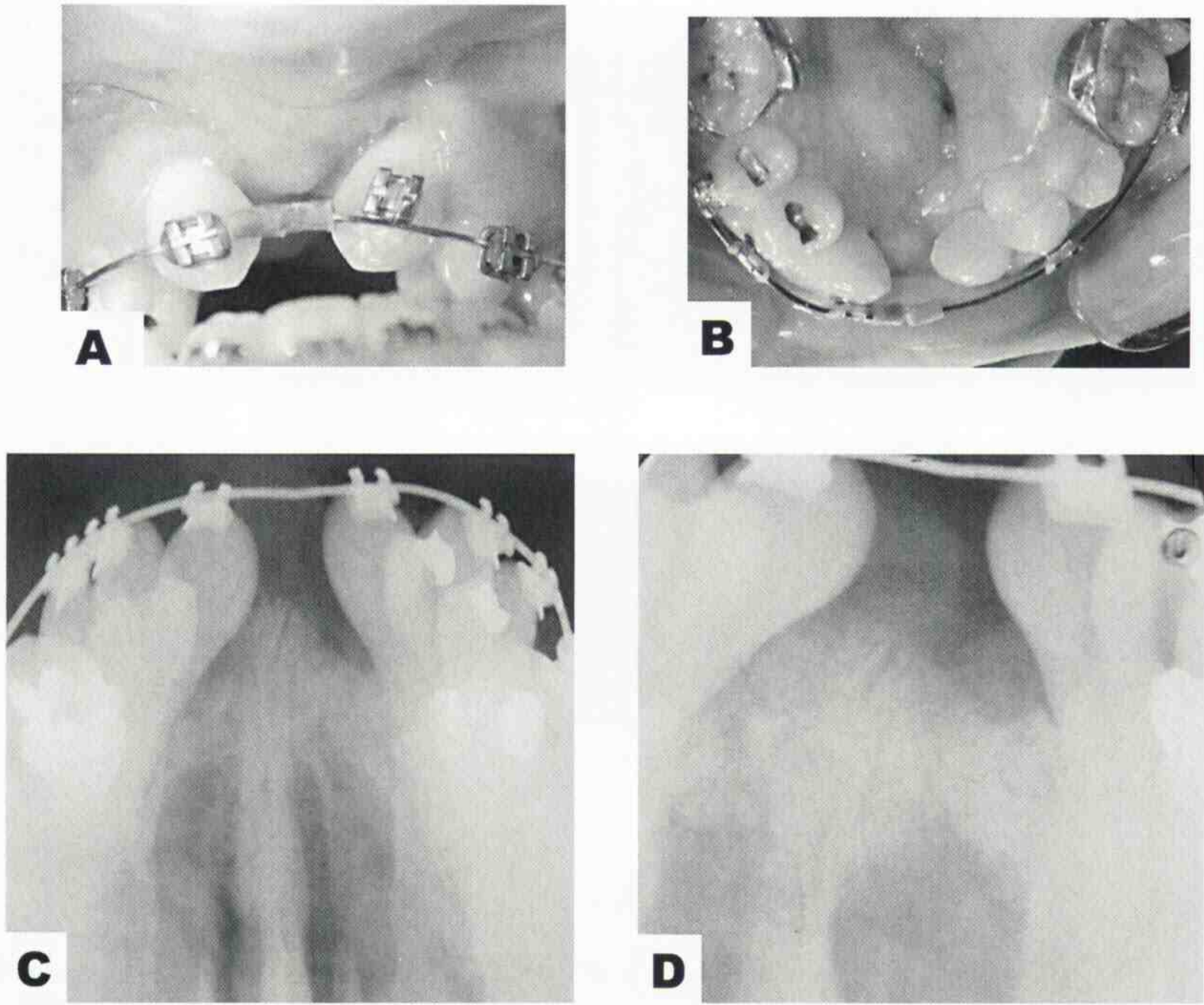

FIGURE 3. Alveolar bone graft for the right side. The right and left side were grafted in separate operations that were 4 months apart. A: Anterior dental photograph taken 6 months after the second side was grafted. B: Occlusal photograph taken 6 months after second side was grafted C: Occlusal radiograph taken 6 months after second surgery. D: Periapical radiograph taken 6 months after second surgery.

hourglass contour of the premaxilla. The nasal roof was established by the suturing of the developed flaps from the premaxilla and the lateral segment. A 1.0-mm bur hole was made in the most anterior, inferior part of the premaxilla and the orthodontic screw was inserted. The orthodontic bracket at the head of the screw was then fastened to the rectangular wire with stainless steel ligature wires so that the premaxilla and the lateral segments were completely stable. Corticocancellous bone from the patient's iliac crest was packed against the exposed bone and into the developed envelope in the usual manner, and the buccal finger flap was sutured to place using 4-0 chromic sutures. To avoid compromising the blood supply to the precarious premaxilla, only one side was grafted at this surgery (lino et al., 1998). The screw remained in place for both surgeries and for 4 months following each surgery.

After 3 months, an occlusal radiograph showed good height of grafted bone in the left side of the cleft. The patient underwent the mirror-image surgery to graft the right side 4 months after the left side was grafted. The finger flaps were raised from mucosa pointing in opposite directions; in other words, the flaps did not overlap and compromise their vascularity. After the second surgery, there was good contour of the alveolar ridge suggesting adequate width to the bone graft (Fig. $3 \mathrm{~A}$ through $3 \mathrm{C}$ ). Occlusal radiographs confirmed the presence of an alveolar bone graft of the right side of the cleft with good height of grafted bone (Fig. 3D). Eventually, the patient will require a LeFort 1 maxillary advancement and prosthetic restorations to restore her occlusion. If the bone grafts were successful, dental implants and supporting surgeries would be considered for replacing her missing maxillary dentition. The bilateral soft tissue flaps helped to eliminate the need for a tongue flap to reduce or close the initial palatal fistula (Fig. 3B). In this case, the premaxilla was able to be grafted to the lateral segments despite marked mobility and the absence of potentially stabilizing teeth.

\section{Discussion}

Many different methods for stabilizing cleft segments have been reported in the literature. The most common techniques 
utilize orthodontic brackets, archwires, or palatal expansion devices. Less common methods include acrylic splints, periodontal dressing, and intersegment pins or wires (Daw et al., 2001). In this case, the premaxilla was small and fragile and could support only one vertical screw placed in the midline. The top of the screw had an orthodontic bracket slot with which to engage a 0.018 -inch rectangular wire. Surgical screws without the orthodontic screw head could also be used; however, the screw would have to be locked onto the archwire with dental cold-cure acrylic or light-cure composite restoration material to immobilize the screw. These types of materials were not needed because screw engagement and wire ligation were sufficient to completely immobilize the premaxillary segment. It should be noted that with this treatment, the premaxilla was attached at two points: the septopremaxillary junction and premaxillary ligation to the archwire via the orthodonticsurgical screw.

Every alveolar cleft is different. Although most alveolar clefts can be managed using orthodontic wires and expanders, the edentulous premaxilla cannot be stabilized with orthodontic brackets and archwire. The screw and wire technique was used instead of the intersegment pins because the segment is more stable and does not incur the risk of placing a pin into dental roots. In this case, the premaxilla did not have enough exposed anatomy to allow a splint to hold the premaxillary position. Moreover, splints have the potential for allowing dental caries to develop under the acrylic surface or periodontal dressing in the area of the lateral segments if oral hygiene is inadequate. Another alternative is to not graft the cleft site. This particular patient will need a LeFort 1 maxillary advancement, so it was advantageous to graft the segments prior orthognathic surgery. Otherwise, the segments would be less stable and require bone grafts at the time of segmental advancement. Other suggested options included the use of a titanium mesh and cancellous bone that would be packed against the denuded premaxillary bone. This treatment was considered risky and a last option because the exposure of the entire surface of the premaxillary bone could compromise the periosteal blood supply and devitalize the premaxillary segment. More extensive surgeries such as a tongue graft were also avoided by using the described approach. In the event that the bone graft failed, we were prepared to treat this patient like a patient who had a premaxilla excised at the time of the primary surgeries. In the past, we have treated three immigrant patients who had their premaxillae excised in their native countries. In these cases, grafting the premaxillary space with cortical and medullary bone from the iliac crest was not successful. Presumably, the blood supply and amount of soft tissue for flap coverage were compromised. Instead, the maxillary canines in the lateral segments were endodontically treated and prepared as overdenture abutments to accept copings and a connecting bar. Sectional partial dentures were made to clip onto a bar spanning between the canines to replace the missing teeth and provide soft tissue support to the upper lip. Although the prosthodontic replacement of the premaxilla was considered for the patient in this case report, it was felt that a successful bone graft could improve her chances for a future orthognathic surgery and give her the option of having dental implants in the future.

The edentulous premaxilla is a rare clinical entity, and our experience is limited to this one case.

Acknowledgments. We wish to thank Dr. Jae-Seung Kim for supplying the orthodontic screws for this surgical procedure. The OSAS self-drilling screw system was provided by Epoch Medical, Seoul, Korea.

\section{REFERENCES}

Abyholm F, Bergland O, Semb G. Secondary bone grafting of alveolar clefts. Scand J Plast Reconstr Surg. 1981;15:127-140.

Boyne PJ, Sands NR. Secondary bone grafting of residual alveolar clefts. $J$ Oral Surg. 1972;30:87-92.

Daw JL, Pavel PK, Kang N-H,Yamashiro D, Morales L. Stabilization of the premaxilla after setback: a discussion of methods. Presented at the 58th Annual Meeting of the American Cleft Palate-Craniofacial Association; Minneapolis, Minnesota; 2001.

Dempf R, Telzrow T, Kramer FJ, Hauseman JE. Alveolar bone grafting with complete clefts: a comparison study between secondary and tertiary bone grafting. Cleft Palate Craniofac J. 2002;39:18-25.

lino M, Sasaki T, Kochi S, Fukuda M, Takahashi T, Yamaguchi T. Surgical repositioning of the premaxilla in combination with two-stage alveolar bone grafting in bilateral cleft lip and palate. Cleft Palate Craniofac J. 1998;35: 304-309. 
Copyright of Cleft Palate Craniofacial Journal is the property of Alliance Communication Group and its content may not be copied or emailed to multiple sites or posted to a listserv without the copyright holder's express written permission. However, users may print, download, or email articles for individual use. 